\title{
COVID-19 coronavirus: Aseguramiento de la calidad en servicio de distribución a domicilio de carne procesada en la ciudad de Ambato
}

Coronavirus COVID-19: Quality assurance in the home delivery service of processed meat in the city of Ambato

Manolo Alexander Córdova Suárez. ${ }^{1}$, Luis Javier Tapia Vasco. ${ }^{2}$, Zoila Eliana Zambrano Ochoa. ${ }^{3} \&$ Maritza Tatiana Chaglla Cango. ${ }^{4}$

Recibido: 25-05-2020 /Aceptado: 19-06-2020 / Publicado: 03-07-2020

\begin{abstract}
.
DOI: https://doi.org/10.33262/concienciadigital.v3i3.1344
\end{abstract}

This research carries out a feasibility study for the implementation of quality assurance measures in the production, marketing and logistics of meat distribution in the city of Ambato, using doorto-door motorized transport in a COVID-19 pandemic period. To establish the changes in the initial process flow, we began by determining the activities, cycles, participants and task execution times by carrying out a work study. Then, a quality assurance analysis was carried out to determine the activities necessary to cover customer satisfaction, considering traceability as priority aspects, basic biosafety measures due to the exposure of workers to environments contaminated by COVID-19 determined by the standard. INEN 1338: 2012 and regulation ARCSA-DE-067-2015-GGG. Next, a process flow diagram was structured considering: macro process, process, inputs, outputs and managers with the list of records necessary to achieve competitive improvement and finally evaluate it with the requirements of the ISO 9001: 2015

1 Department of Food Science and Engineering, Technical University of Ambato, Ambato-Ecuador, ma.cordova@uta.edu.ec

2 Department of Food Science and Engineering, Technical University of Ambato, Ambato-Ecuador, lj.tapia@uta.edu.ec

3 Faculty of Agroindustrial Engineering, Technical University of Cotopaxi, Latacunga-Ecuador, zoila.zambrano@utc.edu.ec

4 Department of Food Science and Engineering, Technical University of Ambato, Ambato-Ecuador, marytatys1992@gmail.com 
standard. Growth was achieved in the following aspects: organizational context by $7 \%$, leadership by $8 \%$, planning by $12 \%$, Support by $6 \%$, operation $13 \%$, performance evaluation $6 \%$ and improvement by 11\% according to the ISO 9001: 2015 standard in extreme working conditions in Ambato by COVID-19. Higher values are observed in items related to production planning.

Keywords: Biological risks, Quality assurance, competitive advantage, COVID-19.

\section{Resumen.}

Esta investigación realiza un estudio de factibilidad para la implementación de medidas de aseguramiento de la calidad en la producción, comercialización y logística de distribución de carne en la ciudad de Ambato, utilizando transporte motorizado puerta a puerta en período de pandemia por COVID-19. Para establecer los cambios en el flujo de proceso inicial se inició determinando las actividades, siclos, participantes y tiempos de ejecución de tareas realizando un estudio de trabajo. Luego se realizó un análisis de aseguramiento de la calidad para determinar las actividades necesarias para cubrir la satisfacción del cliente, considerando como aspectos prioritarios la trazabilidad, medidas básicas de bioseguridad por la exposición de los trabajadores a ambientes contaminados por COVID-19 determinados por la norma INEN 1338:2012 y el reglamento ARCSA-DE-067-2015-GGG. Seguidamente se estructuró un diagrama de flujo de proceso considerando: macroproceso, proceso, entradas, salidas y responsables con la lista de los registros necesarios para lograr la mejora competitiva y finalmente evaluarlo con los requisitos de la norma ISO 9001:2015. Se consiguió un crecimiento en los aspectos: contexto de organización en un 7\%, liderazgo un $8 \%$, planificación un 12\%, Apoyo un 6\%, operación 13\% evaluación del desempeño 6\% y mejora 11\%según la norma ISO 9001:2015 en condiciones extremas de trabajo en Ambato por COVID-19. Se observa valores más altos en los items relacionados a la planificación de la producción.

Palabras claves: Riesgos biológicos, Aseguramiento de la calidad, ventaja competitiva, COVID-19.

\section{Introducción.}

El COVID-19 es un nuevo coronavirus detectado en humanos a fines de 2019. Su aparición ha provocado un gran brote en China y actualmente se ha extendido por todo el globo terráqueo causando problemas en la economía y la adaptación de las personas a esta nueva manera de trabajo en condiciones extremas por su presencia.(Salzberger, Glück, \& Ehrenstein, 2020). La pandemia de COVID-19 también provocó un pánico económico en marzo de 2020. Aunque los países desarrollados con sus fondos de reservas monetarios respondieron al pánico expandiendo sus activos del balance para recuperar los mercados de valores (Sunder, 2020), los países latinoamericanos para recuperar sus economías se vieron en la necesidad de regresar a una manera de trabajo en condiciones extremas por presencia de riesgo biológico con solo medidas de distanciamiento básicas. 
En el mundo en desarrollo, la economía informal puede representar hasta el 80\% de la población donde tanto el empleo informal como las empresas informales son especialmente vulnerables al impacto económico inducido por la pandemia. (Narula, 2020). Los negocios como el de comercio de carne en países de Sudamérica sobreviven con solo el ingenio y la voluntad de sacar adelante familias necesitadas y golpeadas por los efectos negativos d los efectos de la Pandemia por COVID-19.

La gestión actual de la prevención de riesgos laborales se ajusta a la situación excepcional causada por el COVID-19 ello, toma especial importancia con la necesidad de ser rigurosos en las maneras de trabajo que requieren medidas de bioseguridad que garanticen la higiene e inocuidad de los alimentos y salvaguarden la salud de proveedores y compradores en la cadena de suministro.

Esta necesidad de relacionar técnicas que incluyan a los proveedores, comerciante o productores y clientes exige la actuación de una manera planificada con el uso de herramientas de aseguramiento de la calidad que mitiguen el nivel de riesgo biológico y logre una ventaja competitiva a los negocios formales e informales que ejecutan sus actividades en este periodo complicado de vida. La necesidad de reformar el sistema de actuación del empresario (Matsumoto Nishizawa, 2014) ayuda en evitar gastos innecesarios causados por costos sombra (Batz \& Blackman, 2010) especialmente en actividades que son empíricas y que pasan de generación a generación (Fernández, Ruíz, Ruíz, \& Ramón, 2012) con la resistencia al cambio propia de los dueños de los negocios y la incertidumbre de los compradores por el manejo, manipulación de la carne procesada a domicilio, influyen directamente en la disminución de las ventas y pérdidas económicas significativas (Pinzón, 2020).

Las técnicas de aseguramiento de la calidad son extensas (Zapata \& Tejeda, 2009) y su determinación se estima con la interacción de lo necesidad de los clientes como proceso de salida y los proveedores de los procesos predecesores. Este análisis genera herramientas básicas de aplicación a cualquier nivel económico con el uso de documentos relacionados a su procesos principal como son: registros, hojas de verificación, formatos de seguimiento y control entre otros que al asignar responsabilidades en un diagrama de flujo de proceso establecen cumplimento de algunos de los ítems de la norma ISO 9001:2015 que es prioritaria para la satisfacción del cliente (Borja, Chaguay, Bayas, \& Flores, 2017).

Las medidas correctivas que establece este trabajo hace relevante 1 compromiso de los productores del bien o servicio y establece medidas básicas que si son utilizadas con disciplina pueden generar mejores ganancia e ingreso en empresas que realizan actividades relacionadas en tiempos de pandemia por COVID-19 en Ambato Ecuador.

\section{Metodología.}

Materiales y Equipos. 
Los materiales y equipos que se utilizan en la fabricación, manipulación y distribución de la carne procesada son: a) frigoríficos, b) camión repartidor, c) canal de lavado y entre los materiales utilizados están; a) carne procesada, b) agua. Además de instrumentos menores de cocina y material de embalaje propio de este negocio.

\section{Normas de fabricación de alimentos INEN 1338:2012}

Para garantizar las condiciones de inocuidad en la manipulación de alimentos se hiso énfasis en la norma INEN 1338:2012 (Moreira, Solórzano, \& López, 2019) que trata sobre los Productos cárnicos crudos, curados- manipulados y productos cárnicos pre cocidos y cosidos además de algunas otras definiciones relacionadas.

\section{Normas de fabricación de alimentos $\mathrm{N}^{\circ}$. ARCSA-DE-067-2015-GGG.}

Para incluir los procesos de regulación, vigilancia y control sanitario de productos de uso y consumo humano que circulan en el país y de los establecimientos sujetos a vigilancia sanitaria se hiso referencia a la norma ARCSA-067-2015-GGG. Que trata de directrices de inocuidad básicos a ejecutar, con excepción de aquellos prestadores de servicios de salud garantizando las condiciones de procesamiento y reduciendo el riesgo sanitario de productos alimenticios procesados (Coronado \& Sebastián, 2019)

\section{Estudio de Trabajo.}

Para establecer los elementos a controlar con las herramientas de calidad se realizó un estudio de trabajo (Freivalds, 2014) que se centró en establecer los elementos y variables del procesos de estudio siguiendo los siguientes premisas: a) registrar por observación directa de campo cuando ocurren las acciones, b) examinar los hechos con espíritu crítico para saber que se hace en realidad, como se hace y con qué medios, quién realiza las acciones y porqué, c) ordenar las acciones en un gráfico que sirva de guía para un diagrama más estructurado de flujo de procesos en el negocio de comercialización de carne procesada. No se determinó tiempos de ejecución de actividades ni tiempos muertos ya que no es un estudio de tiempos y movimientos además no se consideró ubicación en planta de las estaciones de trabajo ya que no existe restricción de tiempo ni eficiencia.(Criollo \& Magaña, 2005)

\section{Aseguramiento de la Calidad.}

Una serie de esquemas relacionados al aseguramiento de la calidad incluyen instrucciones muy detalladas para realizar el proceso de producción, manipulación y logística de entrega de la carne procesada, por ello fue preciso incrementar herramientas de calidad que permite detectar la utilización de parámetros tecnológicos, físicos, químicos, microbiológicos, nutricionales y sensoriales y lograr que un alimento sea sano y sabroso con el objetivo de proteger al consumidor.(Wotton \& Wilkins, 2009) 
Para establecer los elementos a controlar con las herramientas de calidad se realizó un estudio de trabajo (Carlos, 2009) que se derivó en estas acciones: a) registrar por observación directa cuando ocurren en la distribución, b) listado de verificación de los hechos, como se hace y con qué medios, quién realiza las acciones y porqué, c) ordenar las acciones en un gráfico que sirva de guía para un diagrama más estructurado de flujo de procesos. (Manene, 2011)

\section{Resultados.}

En la Tabla 1 se presentan los resultados correspondientes al estudio del trabajo, considerando condiciones máximas de trabajo.

Tabla 1. Resultado del estudio de trabajo de distribución de carne

\begin{tabular}{|c|c|}
\hline Item & Resultado de la observación \\
\hline Macroprocesos & Producción, ventas, distribución, talento humano \\
\hline Procesos & $\begin{array}{l}\text { Aseguramiento de la calidad, compra y venta de materia } \\
\text { prima, corte de carne fresca, empaquetado y almacenado, } \\
\text { distribución. }\end{array}$ \\
\hline $\begin{array}{l}\text { Actividades de la fabricación y } \\
\text { distribución de carne procesada puesta a } \\
\text { puerta }\end{array}$ & $\begin{array}{l}\text { Publicitar el producto; Compara materia prima; Entregar } \\
\text { kits de bioseguridad a los trabajadores; Capacitar la forma } \\
\text { de entrega del producto; Evaluar a los trabajadores; } \\
\text { Monitorear periódicamente al personal; Desinfectar } \\
\text { planta, equipos y utensilios; Recibir materia prima; } \\
\text { Desinfectar materia prima; Almacenar; Receptar pedidos; } \\
\text { Empacar producto; Desinfectar; Entregar el pedido al } \\
\text { transportista; desinfectar vehículo; Comprobar pedido; } \\
\text { Inspeccionar vehículo y producto, Entregar; Ejecutar } \\
\text { encuesta. }\end{array}$ \\
\hline Entradas del proceso (quien solicita/SGC) & $\begin{array}{l}\text { Sistema de gestión de calidad implementado a la entrega } \\
\text { de alimentos de primera necesidad con el respectivo } \\
\text { protocolo ante COVID-2019 }\end{array}$ \\
\hline Proveedores (del proceso) $*$ & $\begin{array}{l}\text { Manual COVID19 para la industria alimentaria; NTE } \\
\text { INEN 1338:2012; CAC/RCP 58/ 2005; Acuerdo N. } \\
\text { 00126- 202; RESOLUCIÓN ARCSA-DE009- 2020- } \\
\text { LDCL; 23-03-2020 /// Protocolo para establecimientos de } \\
\text { alimentación colectiva }\end{array}$ \\
\hline $\begin{array}{l}\text { Anexos (herramientas de aseguramiento } \\
\text { de la calidad implementadas) }\end{array}$ & $\begin{array}{l}\text { Menú de precios de productos de venta; Contrato de } \\
\text { Trabajo; Registro de proveedores calificados; Registro de } \\
\text { quejas; Medición de satisfacción personal; Lista de } \\
\text { verificación; Registro de selección y capacitación personal } \\
\text { (EPP); Registro de control de máquinas; Registro orden y } \\
\text { limpieza; Registro de entrenamiento personal; Registro de } \\
\text { control de calidad; Registro de eliminación de muestras }\end{array}$ \\
\hline
\end{tabular}


(aceite); Registro de Evaluación de capacitación; Registro de verificación; Registro de control del ambiente

Nota: $\mathrm{SGC}=$ Sistema de Gestión de Calidad. *Los proveedores no se refieren a las empresas que suministran la materia prima. Esta tabla incluye la información necesaria para el estudio realizado, no es un representativo de un estudio del trabajo.

\section{Resultado de implementación de controles de calidad.}

Las medidas de aseguramiento de la calidad se describen en el diagrama de flujo de proceso de la figura 1.

Figura 1. Diagrama de flujo de proceso

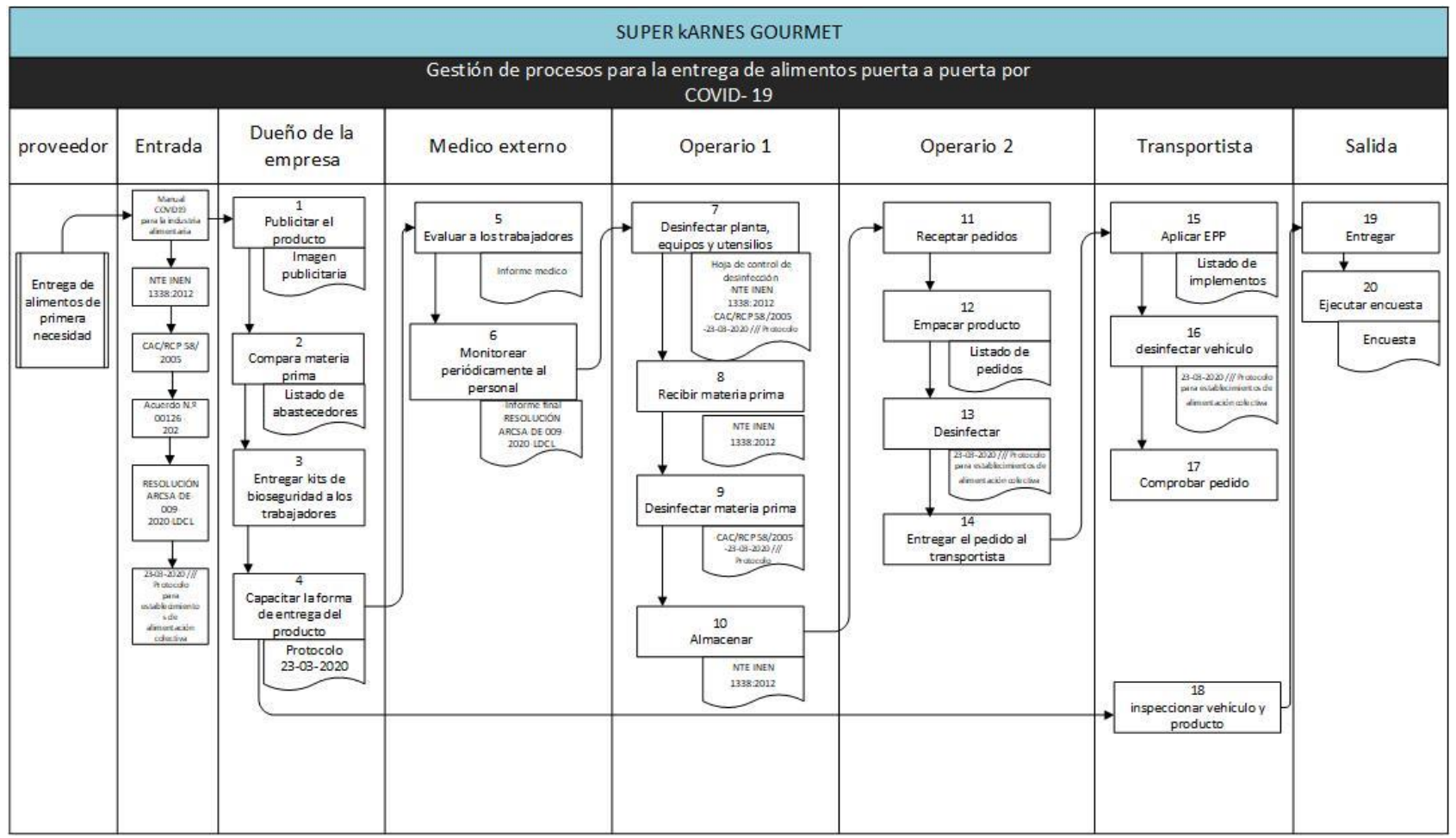

Nota: Los anexos del diagrama de flujo de proceso forman parte del procedimiento de aseguramiento de la calidad completo.

\section{Resultado de la aplicación de la norma ISO 9001:2015}

Para tener una métrica de comparación se realizó la evaluación de los ítems de la norma ISO 9001:2015 en condiciones iniciales (antes de la pandemia por COVID-19) y las posteriores (durante la pandemia por COVID-19) de la aplicación de las medidas de aseguramiento de la calidad. Como no es un trabajo de implementación de la norma los procedimientos relacionados solo se mencionan. 
ISSN: 2600-5859

Vol. 3, N³, p. 486-494, julio-septiembre, 2020

En la Tabla 2 se presenta los porcentajes de cumplimiento de los 4 ítems evaluables de la norma ISO 9001:2015.

Tabla 2. Diagnóstico de evaluación con la aplicación de la NORMA ISO 9001:2015 antes y después de la aplicación de las medidas de aseguramiento de la calidad

\begin{tabular}{lcc}
\hline \multicolumn{1}{c}{ Ítem } & $\begin{array}{c}\text { \% de cumplimiento antes de } \\
\text { la implementación }\end{array}$ & $\begin{array}{c}\text { \% de cumplimiento después } \\
\text { de la implementación }\end{array}$ \\
\hline Contexto de la organización & 0 & 7 \\
Liderazgo & 20 & 28 \\
Planificación & 12 & 24 \\
Apoyo & 12 & 18 \\
Operación & 5 & 18 \\
Evaluación del desempeño & 6 & 10 \\
Mejora & 11 & 22 \\
\hline
\end{tabular}

Nota: tabla de comparación de la aplicación antes y después de la norma ISO 9001:2015, los tresprimeros items son informativos.

\section{Conclusiones:}

- Al implementar las normas como: el manual para la industria alimentaria, NTE INEN 1338:2012, la CAC/RCP 58/2002, el acuerdo No 0012-202, resolución ARCS-DE-0092020-LDCL Y 23-03-2020/// protocolo para establecimientos de alimentos colectiva, para un negocio de entrega a domicilio de carnes crudas, que se sitúa en la ciudad de Ambato en el año 2020, se consiguió un crecimiento en los aspectos: contexto de organización en un $7 \%$, liderazgo un $8 \%$, planificación un $12 \%$, Apoyo un 6\%, operación $13 \%$ evaluación del desempeño 6\% y mejora 11\%según la norma ISO 9001:2015 en condiciones extremas de trabajo en Ambato por COVID-19.

- Se observa valores más altos en los items relacionados a la planificación de la producción.

\section{Referencias bibliográficas.}

Batz, M. B., \& Blackman, A. (2010). Costos de mantenimiento, inertidumbre de precios, y el abandono en la producción de café de sombra: Costa de Oaxaca, México. Gaceta de economía, 16, 151-182.

Borja, G. F. T., Chaguay, L. A. L., Bayas, T. R. F., \& Flores, J. J. C. (2017). El sistema de gestión de calidad en base a las normas ISO 9001 y su relación con los niveles de satisfacción de los clientes. Pro Sciences: Revista de Producción, Ciencias e Investigación, 1(2), 8-11.

Coronado, S., \& Sebastián, G. (2019). DISEÑO DEL MANUAL DE INOCUIDAD ALIMENTARIA BASADO EN LA RESOLUCIÓN ARCSA-DE-067-2015-GGG PARA LA EMPRESA PROSANJO PRODUCTOS SAN JOSÉ CÍA. LTDA. Universidad 
Tecnológica Indoamérica.

Criollo, R. G., \& Magaña, J. P. (2005). Estudio del trabajo: McGraw Hill.

Fernández, M. M., Ruíz, M. T. M., Ruíz, M. R. M., \& Ramón, M. (2012). Seguridad e higiene industrial: gestión de riesgos: Alfaomega Grupo Editor.

Freivalds, A. (2014). Ingeniería industrial de Niebel: métodos, estándares y diseño del trabajo: McGraw-Hill Interamericana Editores, SA de CV.

Manene, L. (2011). Los diagramas de flujo: su definición, objetivo, ventajas, elaboración, fases, reglas y ejemplos de aplicaciones. Recopilado el, 22, 09-18.

Matsumoto Nishizawa, R. (2014). Desarrollo del Modelo Servqual para la medición de la calidad del servicio en la empresa de publicidad Ayuda Experto. Revista Perspectivas(34), 181209.

Moreira, H. J., Solórzano, R. E. B., \& López, P. I. G. (2019). LA EVALUACIÓN DE PROCEDIMIENTOS OPERATIVOS ESTANDARIZADOS Y DE SANEAMIENTO EN EL FAENAMIENTO DE CERDOS. Revista ESPAMCIENCIA ISSN 1390-8103, 10(2), 58-62.

Narula, R. (2020). Policy opportunities and challenges from the COVID-19 pandemic for economies with large informal sectors. Journal of International Business Policy, 1-9.

Pinzón, E. M. R. (2020). Colombia: Impacto económico, social y político de la COVID-19. Análisis Carolina(24), 1.

Salzberger, B., Glück, T., \& Ehrenstein, B. (2020). Successful containment of COVID-19: the WHO-Report on the COVID-19 outbreak in China: Springer.

Sunder, S. (2020). How did the US stock market recover from the Covid-19 contagion? : Springer.

Wotton, S., \& Wilkins, L. (2009). Influencia del procesado y bienestar sobre la calidad de la canal y la carne del broiler. VIII Jorn Técn Intern Avicult de Carne.

Zapata, G., \& Tejeda, I. (2009). Impactos del aseguramiento de la calidad y acreditación de la educación superior. Consideraciones y proposiciones. Calidad en la Educación(31), 192209.

\section{[DDigital}


Vol. 3, N³, p. 486-494, julio-septiembre, 2020

\section{PARA CITAR EL ARTÍCULO INDEXADO.}

Córdova Suárez, M. A., Tapia Vasco, L. J., Zambrano Ochoa, Z. E., \& Chaglla Cango, M. T. (2020). COVID-19 coronavirus: Aseguramiento de la calidad en servicio de distribución a domicilio de carne procesada en la ciudad de Ambato. ConcienciaDigital, 3(3), 486-494. https://doi.org/10.33262/concienciadigital.v3i3.1344

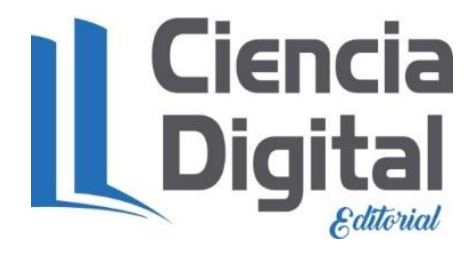

El artículo que se publica es de exclusiva responsabilidad de los autores y no necesariamente reflejan el pensamiento de la Revista Conciencia Digital.

El artículo queda en propiedad de la revista y, por tanto, su publicación parcial y/o total en otro medio tiene que ser autorizado por el director de la Revista Conciencia Digital.
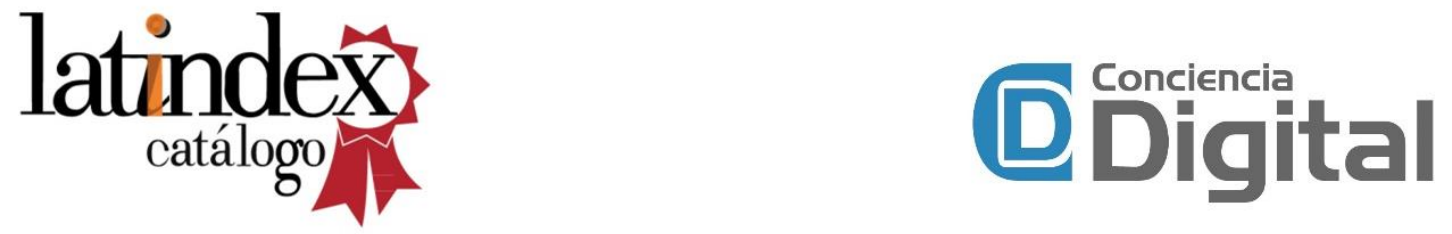